\title{
Comparative Study of Structure, Morphology and Thermal Properties of Allo and Cotton Fibers on Chemical Modification
}

\author{
Netra Lal Bhandari* \\ Department of Chemistry, Tri-Chandra Multiple Campus, Tribhuvan University, Kathmandu, \\ Nepal \\ E-mail:nlbhandari@yahoo.com
}

\begin{abstract}
Nepal is very rich in biodiversity due to the variation on land altitude and climate. Plant fibers are mainly composed of cellulose, hemicellulose and lignin. This paper explains the chemical and morphological changes on plant fibers after chemical treatment such as mercerization and bleaching. Mercerization of fibers was carried out by caustic soda and bleached by hydrogen peroxide or sodium chlorite. The effect of chemical treatment on structure, morphology, thermal, and dying properties of allo (Girardinia diversifolia) and cotton (Gossypium arboretum) fibers were compared. Fibers were characterized by Fourier transform infrared (FTIR) spectroscopy, scanning electron microscopy (SEM), optical microscopy $(O P)$ and thermogravimetric analysis (TGA). Results show that allo fibers have better mechanical and thermal properties than cotton fibers. Further, treated allo fibers are found to be thermally more stable than raw fibers.
\end{abstract}

Keywords: Plant fibers, cellulose, fiber treatment, cotton fibers.

\section{Introduction}

Nepal is blessed with a large variety of flora including wide varieties of fibers yielding plants due to its wide geographical and climatic variations. Natural fibers have been used in the textile industry since a long time ${ }^{[1]}$. Fibers can be extracted from the bark of some plants like ramie, jute, nettle, hemp, from stem like banana, bamboo, from leaf such as palm, sisal, from husk such as coir and from seed such as cotton ${ }^{1,2}$. Different types of fabrics are developed from fibers which are used in textile industry for the preparation of different types of clothes. Cotton, jute, linen and silk are also used in textile industry. Cotton has been worn in India and Egypt for over 5000 years. Cotton was grown by Native Americans as early as 1500 years. Natural fibers are the major sources of readily available biopolymer. Nowadays, the use of natural fiber as filler has been increased due to the number of advantages of natural fibers. These eco-friendly fibers are of low density and with high specific properties ${ }^{1-3}$. Natural fibers are mainly composed of cellulose, hemicelluloses and lignin along with other substances like pectin, wax etc. Normally, plant fiber contains $40-55 \%$ cellulose, $15-35 \%$ lignin, $25-40 \%$ hemicelluloses and others by weight ${ }^{2,4}$. Annually $10^{11}-10^{12}$ tons of cellulose is produced by nature. It is the most important biopolymer on the earth. Cotton fiber is the purest form of natural cellulose which contains only about $5 \%$ of other substances beside cellulose. The chemical composition of natural fibers varies according to the species, age, climate, soil conditions and its geographical distribution ${ }^{[3-6]}$.

\footnotetext{
* Corresponding author
} 
The essential component of all natural fiber is cellulose. Structurally, cellulose is a linear polymer of D-anhydro glucopyranose units linked together by $\beta$-(1-4)-glucosidic bonds. The degree of polymerization (DP) of cellulose is found to be 800 to 10,000 . Hemicellulose is branched polymer which is composed of various five and six carbon sugars, the molecular weight of hemicelluloses is less than that of cellulose ${ }^{[1,6]}$. They consist of a group of polysaccharides (except pectin) which still remain associated with cellulose even after the removal of lignin. Lignin is an amorphous, crosslinked polymer network consisting of an irregular array of variously bonded hydroxyl and methoxy substituted phenyl propane units. These are soluble in sodium, hydroxide and hence lignin can be removed to some extent by alkali treatment and bleaching ${ }^{5,6}$. Lignin is hydrophobic in nature. The moisture content of the fibers depends on the content of non-crystalline parts and void content of the fiber which amounts up to $10 \mathrm{wt} . \%$ under standard conditions. The hydrophilic nature of natural fibers influences the overall mechanical properties as well as other physical properties of the fiber itself ${ }^{6-8}$. Various chemical modifications and treatments such as graft copolymerization, mercerization, silane treatment, acetylation, benzoylation, permanganate treatment etc. have been used to overcome the disadvantages associated with natural fibers. Preparation of bio-composting polymer composite material to preserve the environment is also one of the goals of this research. The applications of natural fiber polymer composites are increasing different fields such as carpeting, electrical instrument, packing, sports and many others ${ }^{[6-10]}$.

The aim of this study is to investigate the feasibility of extraction of cellulose fibers from natural fibers such as Allo (Girardinia diversifolia), Bamboo (Bambusa nutans), cotton (Gossypium arboretum) etc. by different procedures and study the changes in their morphology, structure, thermal stability and dying fastness properties. Nowadays, preparation of composite materials by using differently modified fibers as well as the use of fibers in various textile and cloth industries have been in use due to high environmental consciousness.

\section{Experimental Methods}

\section{Chemicals and Materials}

Hydrogen peroxide $\left(\mathrm{H}_{2} \mathrm{O}_{2}\right.$, SD Fine-Chem Ltd., India), Sodium hydroxide ( $\mathrm{NaOH}$, Thermo Fischer Scientific India Pvt. Ltd.), Acetic acid $\left(\mathrm{CH}_{3} \mathrm{COOH}\right.$, Thermo Fischer Scientific India Pvt. Ltd.), Sulphuric acid $\left(\mathrm{H}_{2} \mathrm{SO}_{4}\right.$, Thermo Fischer Scientific India Pvt. Ltd), Formic acid (HCOOH, Thermo Fischer Scientific India Pvt. Ltd) were purchased from chemical suppliers in Kathmandu valley. Different natural fibers from different plants were collected from the village nearby Kathmandu valley and also from suppliers in Kathmandu, Nepal.

\section{Extraction of Plant Fibers}

Raw allo fiber was treated with hydrogen peroxide followed by caustic soda treatment. The fiber was dried at $80^{\circ} \mathrm{C}$ for 10 hours. Cotton fiber was collected from Syangja district of Nepal. It was purely white, pure and is not treated with alkalies.

\section{Characterization of Fibers}

Plant fibers were characterized by the different spectroscopic and microscopic techniques. Thermal and microscopic study of fiber was carried out at Leibniz-Institut für Polymerforschung, Dresden, Germany and Institute of Physics, Martin Luther University, Halle Germany respectively. The Fourier 
transform infrared spectroscopy was carried out in Academy of Science and Technology (NAST), Khumaltar, Lalitpur. The Water absorption and optical microscopy was done in Research Center for Applied Science and Technology (RECAST), Tribhuvan University, Kathmandu.

\section{Results and Discussion}

Figure 1, represents the steps of modification of allo fibers to extract the white allo fibers contacting higher proportion of cellulose from it. The treated allo fibers are purely white in color almost similar to that of the cotton fibers (shown in Figure 1a). Nowadays these fibers are widely used in textile industries as well as in polymer composite manufacturing industries as fillers to make eco-friendly composites. Figure $1 \mathrm{~b}$ shows the simple cotton fibers which are collected directly without any further chemical treatments.
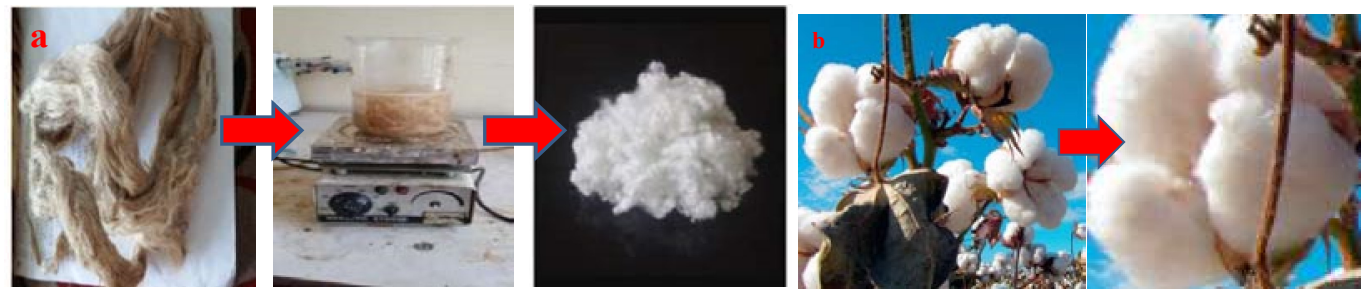

Figure 1: Steps for extraction process of a) treated allo fibers from raw allo fibers and b) cotton fibers

The structural changes in treated allo fibers were studied by Fourier transform infrared (FTIR) spectroscopy and compared with neat allo fibers and cotton fibers as shown in Figure 2.
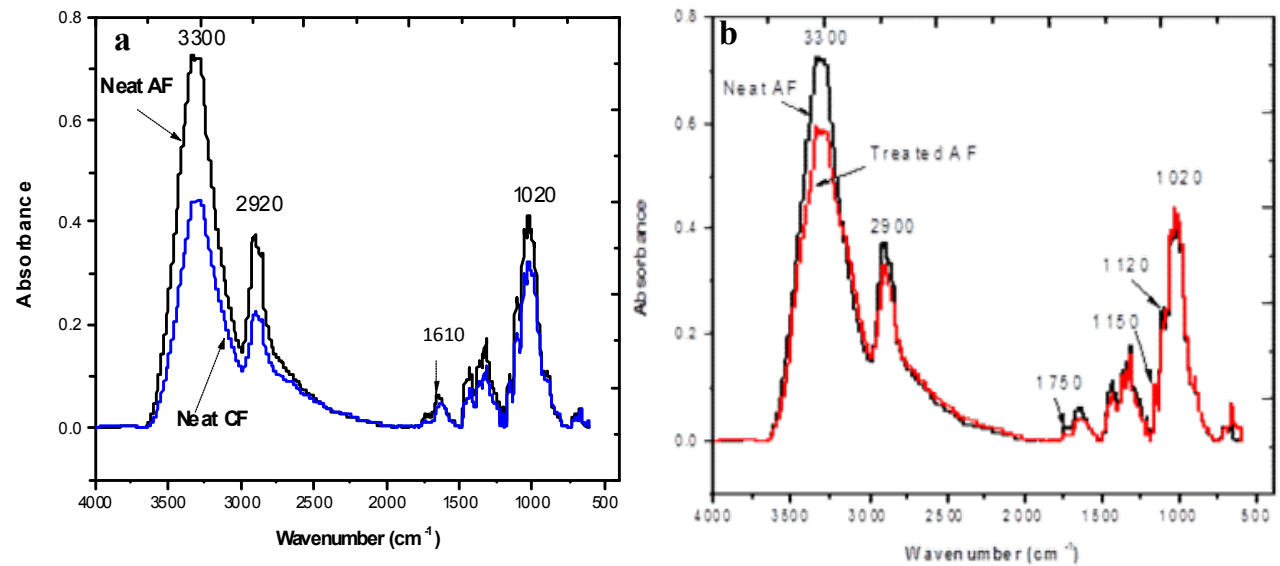

Figure 2: Comparison of FTIR spectra of a) neat and treated allo fiber and b) neat allo fibers and cotton fibers

Figure 2a shows the spectrum of allo fibers which was compared with cotton fibers. It shows that the amount of cellulose in allo fiber and cotton are nearly equal due to similar peak positions in the spectra. Peaks at $3300 \mathrm{~cm}^{-1}$ (-OH stretching), $2920 \mathrm{~cm}^{-1}$ (C-H stretching) and $1020 \mathrm{~cm}^{-1}$ (C-O-C stretching vibrations of pyranose ring) are the characteristic peaks of cellulose in natural fibers ${ }^{[6,7]}$. 

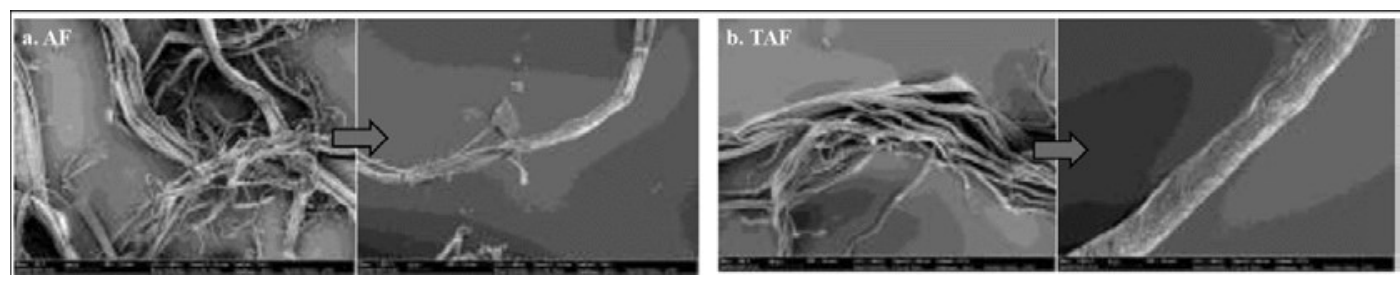

Figure 3: SEM image of a) allo fibers $(A F)$ and b) treated allo fibers (TAF) under low and high magnifications

After chemical treatment of allo fibers, some peaks were disappeared while some of them were reduced suggesting the decrease of lignin and hemicelluloses content. The increase in peak intensity at $3300 \mathrm{~cm}^{-1}$ and $1020 \mathrm{~cm}^{-1}$ and disappearance of peaks at $1510 \mathrm{~cm}^{-1}\left(\mathrm{C}=\mathrm{CH}_{2}\right), 1234 \mathrm{~cm}^{-1}\left(\mathrm{C}-\mathrm{OCH}_{3}\right.$ stretching) and $830 \mathrm{~cm}^{-1}$ (C-O-stretching) represent the increase in cellulose and decrease in phenolic content of lignin and hemicelluloses of fibers ${ }^{[6]}$. The morphological changes after the chemical treatment of allo fiber was also observed by the scanning electron microscopy as shown in Figure 3.

The surface morphology of fibers was studied by scanning electron microscopic analysis. Figure 3 shows the morphological differences in between the neat and treated allo fibers. The figure on right represents the image of single fiber under high magnification. In chemically modified fibers, cellulose microfibrills are linearly aligned and clearly visible with their smooth surfaces which might be due to the delignification during chemical treatment. In neat fibers, the cellulose fibers are in bundles with rough surfaces. The lignin might help to bind these cellulose fibers to form the bundles ${ }^{[6,8]}$. It shows that the chemical treatment helps to improve the surface morphology of natural fibers.
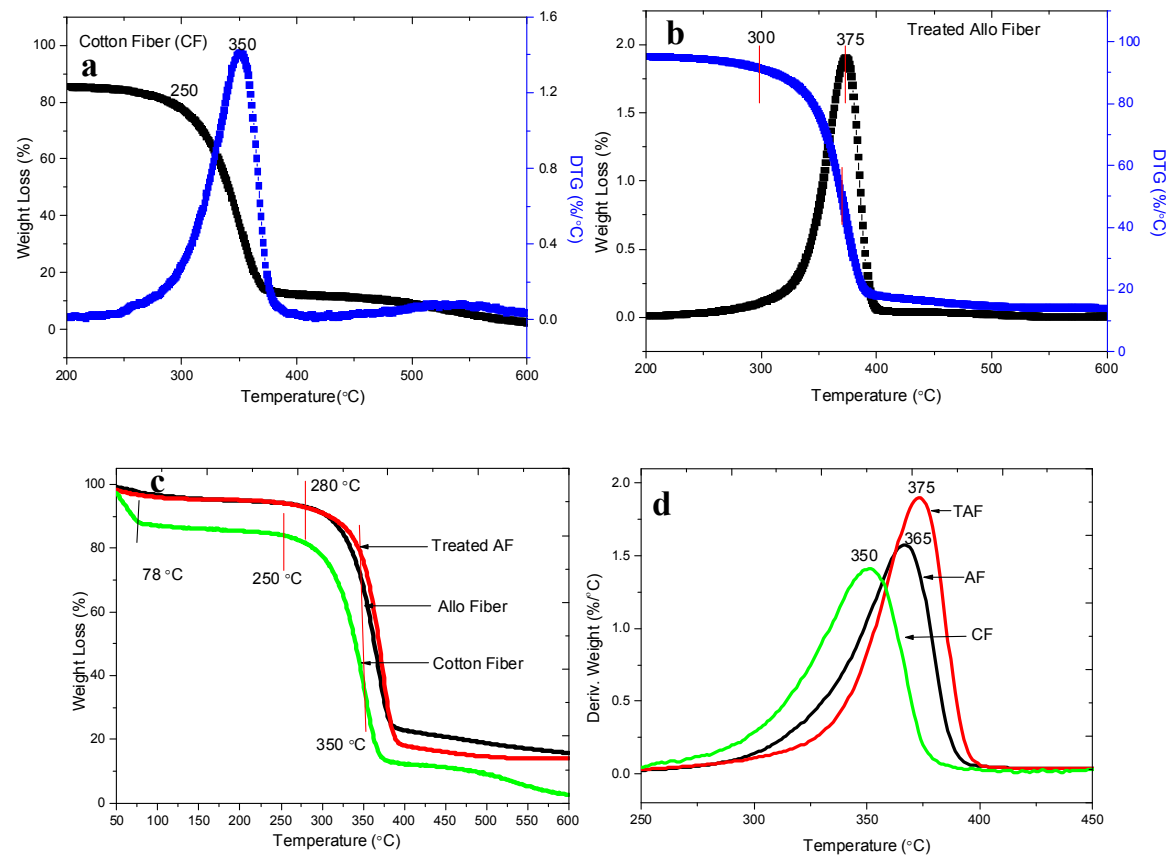

Figure 4: TGA and DTG analysis of allo and cotton fibers

$-40-$ 
Pyrolysis of allo fibers and treated allo fibers were analyzed by thermogravimetric analysis (TGA) and differential thermogravimetry (DTG). The thermal stability of fibers was compared with cotton fibers. Both neat and treated allo fibers were found to be thermally more stable than that of pure cotton fibers as suggested by Figure $4 \mathrm{a}$ and Figure $4 \mathrm{~b}$. The degradation of cellulose in cotton fibers starts from $250{ }^{\circ} \mathrm{C}$ and the maximum pyrolysis occurs at $350{ }^{\circ} \mathrm{C}{ }^{[4-7]}$. Mass loss in allo fibers and treated allo fibers starts at $260{ }^{\circ} \mathrm{C}$ and at $280{ }^{\circ} \mathrm{C}$ respectively. The maximum mass loss of neat and treated allo fibers was observed at $365{ }^{\circ} \mathrm{C}$ and $375{ }^{\circ} \mathrm{C}$ respectively. It shows that treated allo fibers are found to be stabilized by $15^{\circ} \mathrm{C}$ than raw allo fibers and about $25{ }^{\circ} \mathrm{C}$ than cotton fibers. Further the residual mass in treated fiber is $10 \%$ whereas in neat fibers it is about $20 \%$. Allo fibers are thermally more stable than cotton fibers.

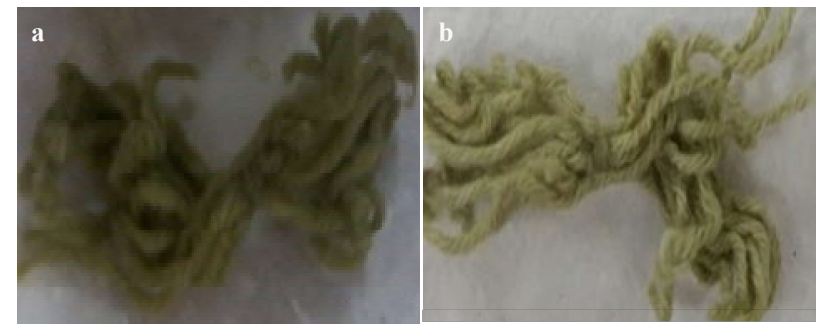

Figure 5: Optical photographs shows light and wash fastness of dyeing of a) cotton fibers (left) and b) treated allo fibers (right) with balsam dye and lemon juice as moderant

Further the dying and wash fastness properties on allo and treated allo fibers were studied by dying the fibers with balsam dyes by using lemon juice as mordant ${ }^{9,10}$. The results are shown in Figure 5 . The figure on left represents the wash fastness on cotton and on right represents the wash fastness on allo after one week coloured under similar conditions. The result shows that the color and color fastness is more prominent and attractive on allo fibers than in cotton fibers.

\section{Conclusions}

Due to the huge biodiversity of Nepal, it would be the best laboratory in the world to study plants and plant fibers. In this study allo fibers are found to be better than cotton fibers. Treated allo fibers are found to be still better than the neat fibers. The morphology, mechanical, thermal as well as the dying fastness properties of plant fibers can be enhanced by simple chemical modification. This idea can contribute strappingly on textile or fabric production and dying industries as well as production of sustainable ecofriendly smart composite materials.

\section{Acknowledgements}

Author is profoundly thankful to Institute of Physics, Martin Luther University, Halle Germany for the SEM analysis and Leibniz-Institut für Polymerforschung Dresden, Dresden, Germany for the thermal analysis of fibers. Recesearch Center for Applied Science and Technology (RECAST), Tribhuvan University, Kirtipur, Kathmandu is also appreciative for the laboratory facilities.

\section{References}

1. S. Fakirov, Handbook of Engineering Biopolymers: Homopolymers, Blends and Composites, Hanser Publishers, Munchi, 2007. 
2. A. K. Bledzki, J. Gassan, Progress in Polymer Science, 1999, 24, 221.

3. S. G. Bogoever-Gaceva, M. Avella, M. Malinconico, A. Grozdanov, G. Genitale, M. E. Errico, Polymer Composite, 2007, 6, 98.

4. V. K. Varsheney, S. Naithani, Green Chemistry and Technology, Springer 2011.

5. A. K. Mohanty, M. Mishra and L. T. Drzal, Journal of Polymers and the Environment, 2002, 10, 19.

6. N. L. Bhandari, S. Thomas, C. K. Das, R. Adhikari, Nepal Journal of Science and Technology, 2012, 13, 95 .

7. H. Yang, R. Yan, H. Chen, D. H. Lee, C. Zheng, Fuel, 2007, 86, 1781.

8. R. Adhikari, N. L. Bhandari, H. H. Le, S. Henning, H.- J. Radusch, G. H. Michler, M-R. Garda, J. M. Saiter, Macromolecular Symposia, 2012, 315, 24.

9. S. Saxena, A.S.M. Raja, Textile Science and Clothing Technology, 2014, 35.

10. N. K. Parajuli, Dissertation: M.Sc. Chemistry-2017, Tri-Chandra Multiple Campus, Tribhuvan University, Kathmandu, Nepal, 2017. 